Bull. Matet. Sa., Vol. 49, No. 1, Februaly 1990, pp. 29-38. O Printed in India.

\title{
Hydrogen induced microstructural variation in diamond and diamond like carbon thin films
}

\author{
V D VANKAR \\ Department of Physics, Indian Institute of Technology, New Delhi 110016, India
}

\begin{abstract}
Hydrogen plays a crucial role in the growth of micro-crystalline diamond (MCD) and diamond like carbon (DLC) thin films grown by plasma assisted chemical vapour deposition (PACVD) processes. It selectively etches graphite phase and helps in stabilizing the diamond phase. The presence of various hydrocarbon species in the plasma and their reaction with atomic. excited or molecular hydrogen on the substrate surface decide the mechanism of dramond nucleation and growth. Several mechanisms have been proposed but the process is still not well understood.

Control of hydrogen and other deposition parameters in the PACVD process leads to deposition of yet another class of materials called diamond like carbon. By varying the wncentration of hydrogen it is possible to produce purcly amorphous carbon films on the one hand and amorphous hydrogenated carbon films (with as high as $60 \%$ hydrogen) on the other. Very hard, optically transparent and electrically insulating films characterize the diamond like behaviour. The proportion of hydrogen and its bonding with carbon or hydrogen in the film can be varied to obtain very hard to very soft films which could be optically transparent or opaque. The microstructure of these films have been investigated by a large number of rechniques. The results show interesting situations.

This paper reviews the work on the role of hydrogen on the growth, structure and properties of MCD and DLC thin films.
\end{abstract}

Keywords. Diamond; hydrogen; microstructural variation; graphite; plasma assisted chemical vapour deposition; amorphous; microcrystalline; epitaxy; insulator; optical properties; supersaturation; nucleation and growth.

\section{MCD thin films}

Extensive research is being carried out, all over the world, on the growth of diamond and diamond like carbon thin films using PACVD techniques employing various hydrocarbon sources. Major breakthroughs in low-pressure diamond deposition technology have been possible mainly due to the addition of hydrogen in the reactive gas mixtures. The best quality microcrystalline diamond (MCD) thin films have been obtained in hydrogen atmosphere containing only small amount (e.g. $1 \%$ or less, of methane) of hydrocarbon in the reaction mixture.

A variety of processes have been used for MCD thin films deposition. These include microwave/rf/dc plasma CVD processes employing methane, acetylene, butane, benzene, acetone, methyl alcohol etc. as the source of carbon. All these hydrocarbon sources contain plenty of hydrogen and it is released during the plasma decomposition process. A large number of radicals and ions are produced in the plasma as a result of reaction between the hydrogen and the dissociation products of the hydrocarbon (Frenklach 1989). Thus, in the $\mathrm{CH}_{4}-\mathrm{H}_{2}$ plasma the chemical species produced are $\mathrm{CH}_{4}^{+}, \mathrm{CH}_{3}^{+}, \mathrm{CH}_{2}^{+}, \mathrm{CH}, \mathrm{C}^{+}, \mathrm{H}_{2}^{+}, \mathrm{CH}_{5}^{+}$and $\mathrm{H}_{3}^{+}$as well as neutrals $\mathrm{CH}_{3}, \mathrm{CH}_{2}, \mathrm{CH}, \mathrm{C}$, $\mathrm{H}$ and the excited species. These active species can react on the substrate surface and lead to nucleation and growth of the diamond microcrystals. At low pressures (atmosphere or below) diamond is metastable, the stable phase being the graphite 
(figure 1). The free energy difference between the graphite and the diamond is only $0.02 \mathrm{eV} /$ atom $(500 \mathrm{cal} / \mathrm{mole})$. As a result in a growth process, diamond phase is formed in a continuous competition with the thermodynamically favoured graphite phase. The probability of nucleation and growth of diamond is negligibly small as compared to that of graphite.

In a typical PACVD process of diamond growth several overlapping elementary processes work together. These may be classified in three groups - (1) dissociation of the hydrocarbon source and the formation of the necessary intermediate species, (2) transport of the reactive species to the substrate surface and the occurrence of the nucleation process and (3) stabilization of the diamond phase on the surface of the growing film. Knowledge of the reaction pathways and the ability to control the rate determining step are crucial for consistent and reproducible film characteristics (Inspector et al 1989).

In addition to the above factors, hydrogen plays a very important role in diamond synthesis and this is due to the preferential etching of graphite in contrast to diamond in hydrogen plasma environment. The relative gasification rates of diamond and graphite are known to be different by orders of magnitude (Setaka 1989). As a result the nucleation and growth of graphite is inhibited due to the presence of atomic hydrogen in the plasma environment. There are numerous variations on this theme. Calculations show that at least some of the experimental data can be rationalized if one assumes that in the presence of atomic hycirogen diamond is kinetically stable relative to graphite (Sommet et al 1989). The second important effect of hydrogen is to satisfy dangling bonds of surface carbon atoms keeping them in the $s p^{3}$ configuration and thus preventing the diamond surface from reconstruction into graphitic $s p^{2}$ or carbynic $s p^{1}$ structures. Thus, for example, Yang et al (1993) have shown formation of monohydride surface structure with one hydrogen atom per surface carbon atom on diamond (100)

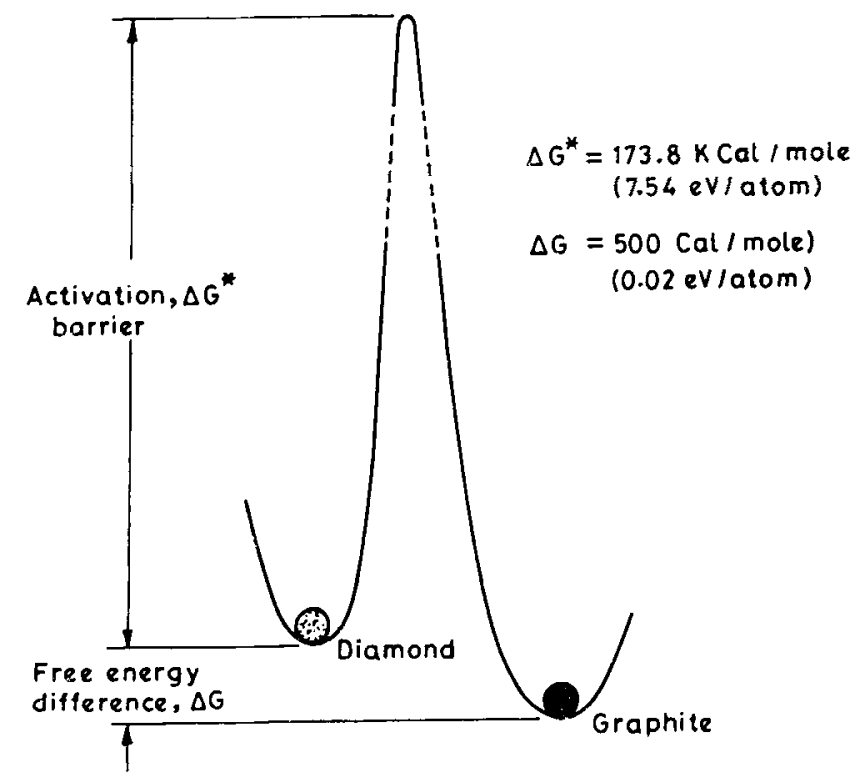

Figure 1. Free energy difference and activation barrier for graphite and diamond phases. 
surface. It was predicted (Pate 1986) that the $(2 \times 1)$ : H monohydride phase is the most stable thermodynamically and is the dominant phase under typical chemical vapour deposition condition (figure 2). Estimates of enthalpies of formation of diamond and graphite surfaces, which have been hydrogenated, yield lower values for diamond than graphite. Enhanced etching of graphite by hydrogen is then viewed as a reflection, in part, of the relative instability of graphite surfaces to gasification (Pepper 1982).

Hydrogen also plays an important role for removal of chemisorbed oxygen from the substrate surface which is invariably present in a deposition process. Struck and D'Evelyn (1993) have found evidence for the existence of $\mathrm{C}-\mathrm{OH}, \mathrm{C}-\mathrm{O}-\mathrm{C}$ and $>\mathrm{C}=\mathrm{O}$ modes on the surfaces of diamond exposed to hydrogen and water vapour. They suggest a potentially important role for surface hydroxyl and oxide species in the surface chemistry and morphological development of diamond films grown by CVD processes.

Inspector et al (1989) have investigated the role of atomic hydrogen on the plasma chemistry in diamond deposition. Their findings suggest that atomic hydrogen is the active intermediate in the dissociation process and high concentrations of atomic hydrogen are required to accelerate the formation of carbonic deposits at the substrate. Secondly, regardless of the starting material, similar $1-2$ carbon atom containing species are produced in the plasma and apparently become the building blocks of diamond film. And, thirdly, the nature of the deposit i.e. the ratio between graphite and diamond content of the film is affected by the processes inducted at the surface of the growing film (Yarbrough and Messier 1990).

The dilution of the hydrocarbon to as much as $1 \%$ by hydrogen is also necessary to inhibit polymerization of the hydrocarbon source in the plasma volume. High concentration of hydrogen also causes quenching effects, such as the reduction of electron

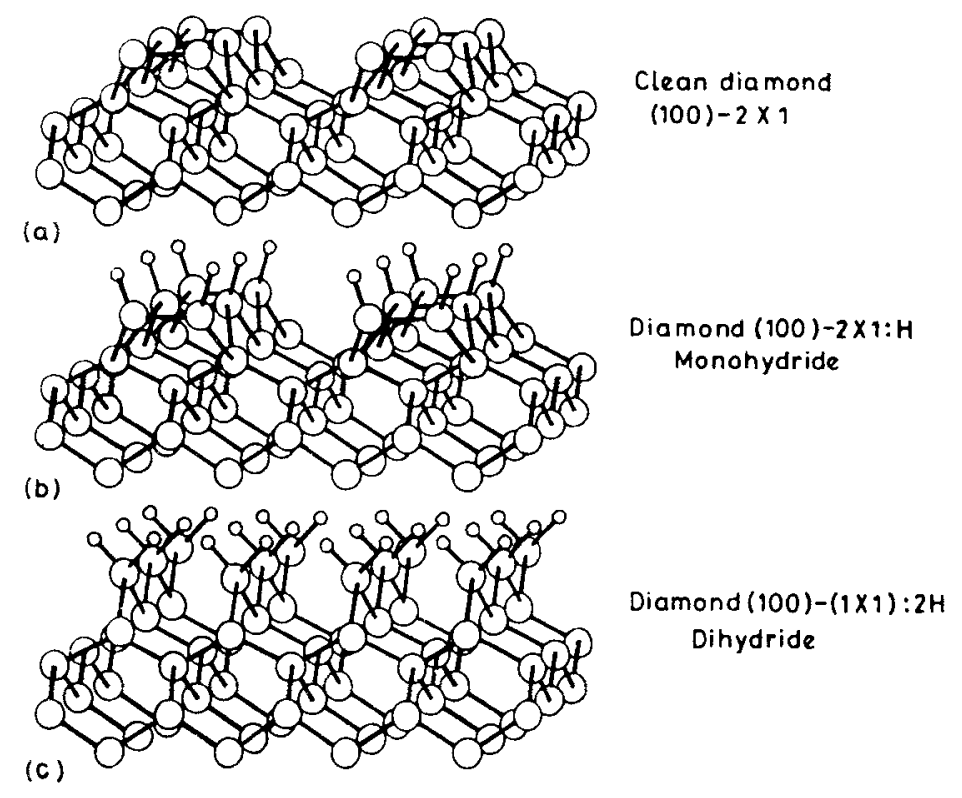

Higure 2. Surface atomic structure of (a) clean diamond $(100\}-\{2 \times 1\}$, (b) the $(100)-(2 \times 1): \mathrm{H}$ monohydride and $(c)$ the $(100)-(1 \times 1): 2 \mathrm{H}$ full dihydride (Pate 1986). 
energy and of the degree of ionization in the plasma. This causes further inhibition of the polymerization processes in a diamond forming plasma. The dissociation of hydrogen and hydrocarbon species introduced in a plasma and subsequent recombination of the ionized species are the competing processes which are responsible for the production of atomic hydrogen or its depletion in the process and thereby control the kinetics of the growth of diamond thin films.

Quantum mechanical calculation of Tsuda et al (1986) to determine the lowest energy path for a proposed mechanism of diamond (111) growth predicts a two-step reaction sequence (figure 3 ). In the first step the diamond surface is covered by a methyl group via either methylene insertion or hydrogen abstraction followed by methyl radical addition. In the second step three neighbouring methyl groups are bound together to form the diamond structure. Frenklach and Spear (1988) proposed an alternative mechanism for the growth of (111) diamond surfaces. The first step is the surface activation by $\mathrm{H}$-atom removal of surface bonded hydrogen. The activated carbon radical then acts as site for adding more carbons to the structure by reacting with acetylene (or other carbon-hydrogen species in the gas plasma). Further addition of two acetylene molecules for one hydrogen abstraction is the next step which regenerates the hydrogen atom and the process continues (Spear 1989). Huang et al (1988) have analysed these models and found that the first step in the process is the removal of a surface bonded hydrogen which requires surface activation, all other subsequent mechanistic steps occur without any activation. Several multistep bond breaking, atom transfer bond-forming processes may be energetically more favourable if they occurred as one simultaneous step rather than as sequential steps. All these

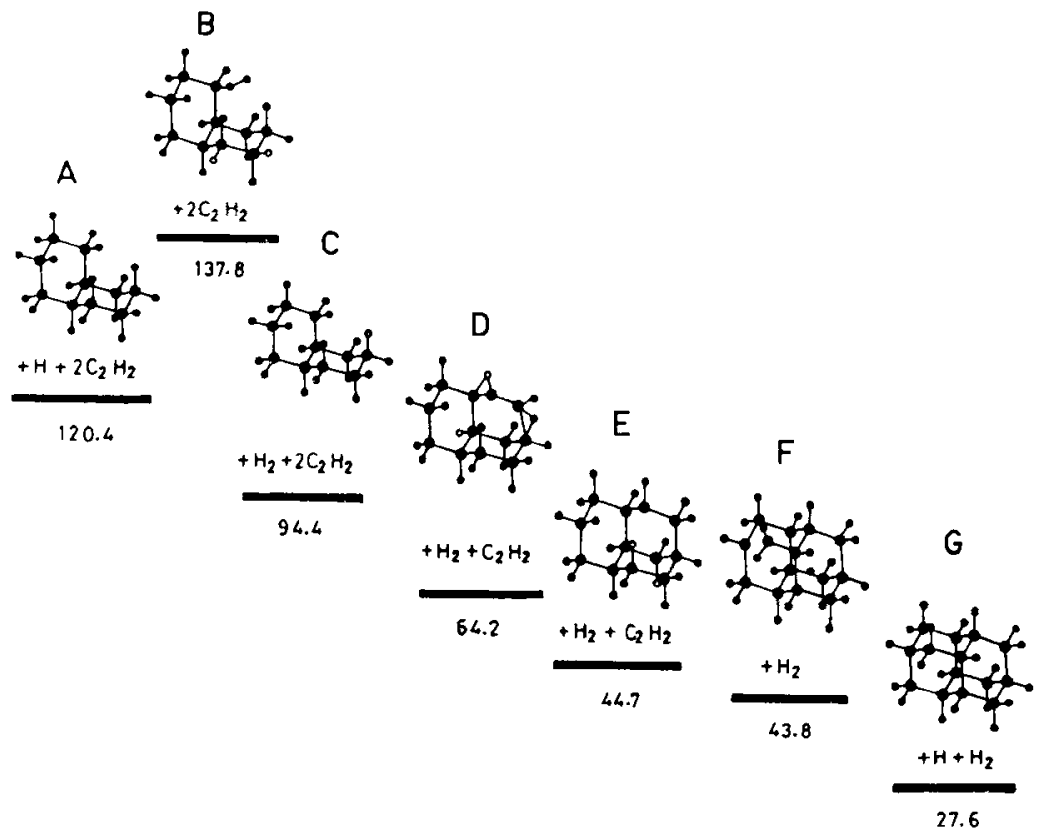

Figure 3. Energy diagram for a possible reaction sequence of epitaxial diamond growth (Huang et al 1988). 
Increasing Hydrocarbon / Hydrogen ratio

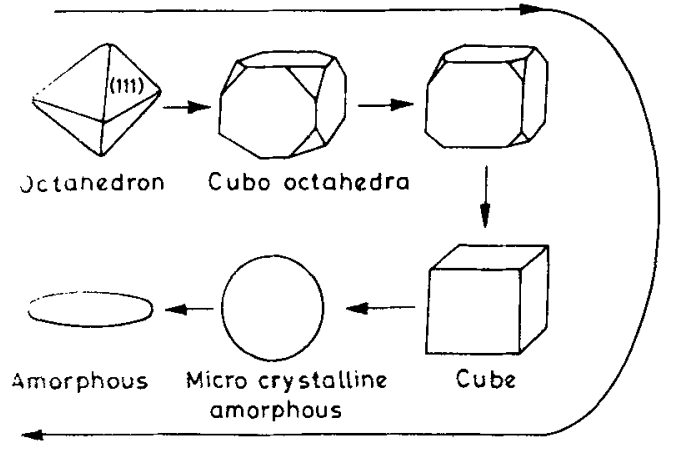

Figure t. Effecl of hydrocarbon to hydrogen ratio on diamond microstructure (Rossi 1992).

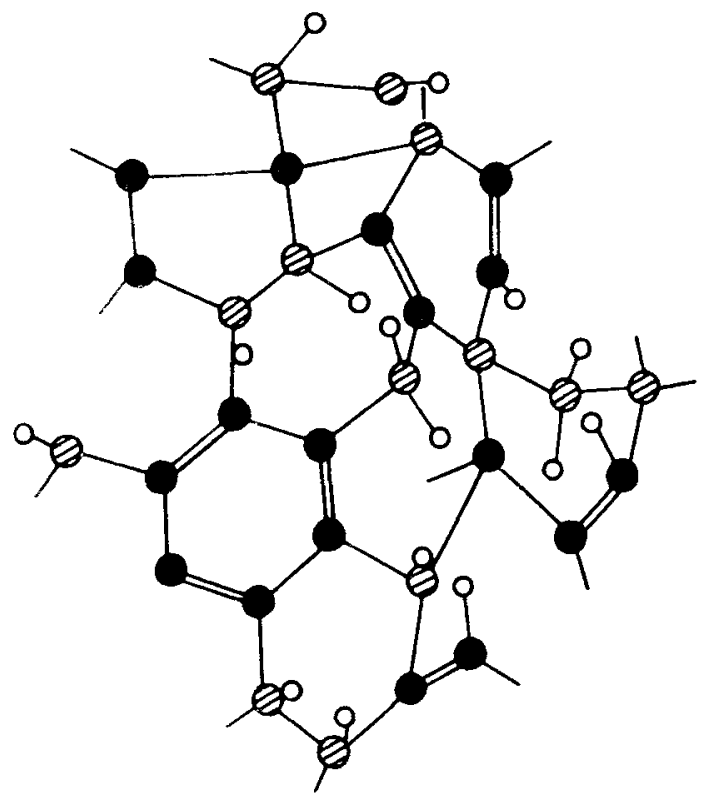

$$
\begin{aligned}
& -S p^{3} C \text { atom } \\
& -S p^{2} C \text { atom } \\
& O-H \text { atom }
\end{aligned}
$$

Figure 5. An ixample of a bonding configuration in DLC thın films.

processes evidently emphasize the importance of hydrogen in the growth of diamond films.

The morphological changes and deposit structure of MCD thin films have been reviewed by Vandenbulcke et al (1991). Different crystal habits can be obtained in MCD 
thin films ranging from spherulatic, polycrystalline shapes and irregular forms to well faceted separate crystals with cubic, cubo-octahedral or octahedral habits showing eventually single and multiple twins, and to polycrystalline layers composed of the preceding forms. The morphology and structure has been shown to depend upon the supersaturation over graphite relative to that over diamond. The partial pressures of $\mathrm{C}$-containing species at the gas-solid interface relative to atomic hydrogen has been found to influence the overall growth of diamond morphology and its ultimate purity in different techniques of deposition (figure 4) (Rossi 1992).

\section{DLC thin films}

DLC films, in contrast to MCD films, are distinguished by amorphous or quasiamorphous arrangement of atoms with surface crystallites whose structures are yet to be determined unequivocally (Robertson 1986). These films contain varied amount of hydrogen bonded to carbon atom either in $s p^{3}$ or $s p^{2}$ or $s p^{1}$ configuration as shown in figure 5 . As a result, soft to very hard, transparent to opaque and conducting to insulating films have been obtained using a wide variety of techniques (Tsai and Bogy 1987). These techniques employ either a hydrocarbon source or a carbon source. Since the process involves bombarding ions of carbon or hydrogen of different energies, a range of properties get generated in such films. Figure 6 illustrates the influence of ion
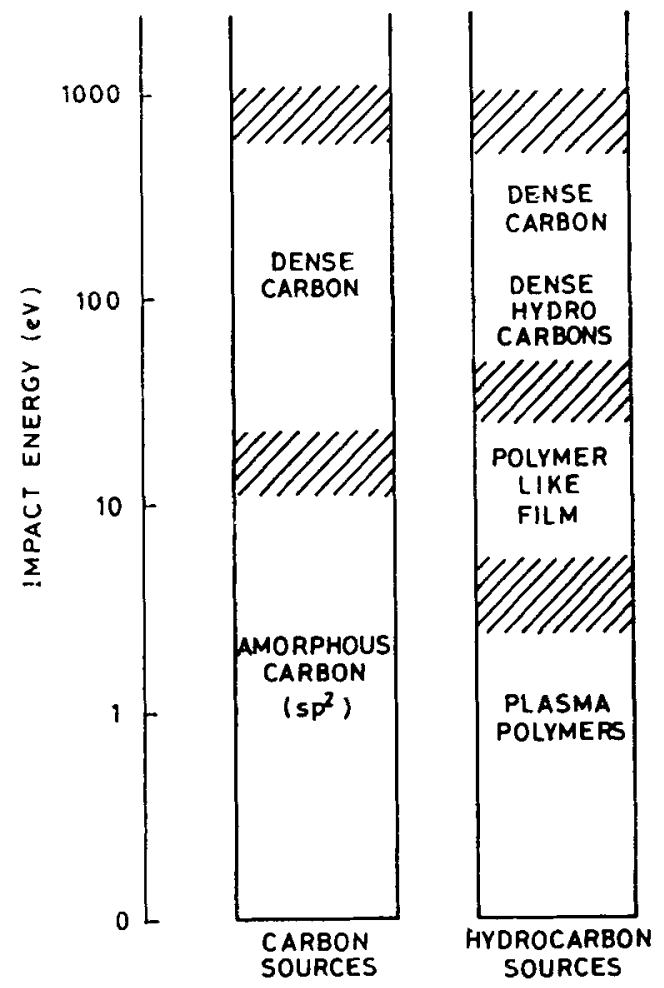

Figure 6. Influence of impact energy on the type of films produced (Angus et al 1986). 
energy on the type of films produced (Angus et al 1986). Plasma polymers contain large amount of hydrogen but dense carbon contains very little hydrogen.

Figure 7 shows the effect of heat treatment on the IR spectrum of a typical DLC thin film. Hydrogen is evolved upon heating these films above $380^{\circ} \mathrm{C}$. Nyaiesh and Nowak (1983), found exothermic transitions at $550^{\circ} \mathrm{C}$ and $750^{\circ} \mathrm{C}$. Dischler et al (1983) have proposed a model for thermally induced changes in DLC films which contain $s p^{3}, s p^{2}$ and $s p^{1}$ bonded hydrogen in the carbon network and existence of hypothetical microvoids in these films (figure 8). It was found that as grown a-C:H films contain about $2 / 3$ of the carbon atoms which are tetrahedrally coordinated $\left(s p^{3}: s p^{2}: s p^{1}=68\right.$ : $30: 2$ ), thermal annealing above $600^{\circ} \mathrm{C}$ converts all the $s p^{3}$ bonded carbon into aromatic $s p^{2}$ rings. Similar results have been obtained by other workers also (Nadler $e t$ al 1984; Couderc and Catherine 1987; Hernandez et al 1989).

Prawer et al (1986) have irradiated DLC films with focussed pulsed laser beams and found that highly insulating $\left(10^{6} \mathrm{ohm} \mathrm{cm}\right)$ DLC layers, transformed into conducting
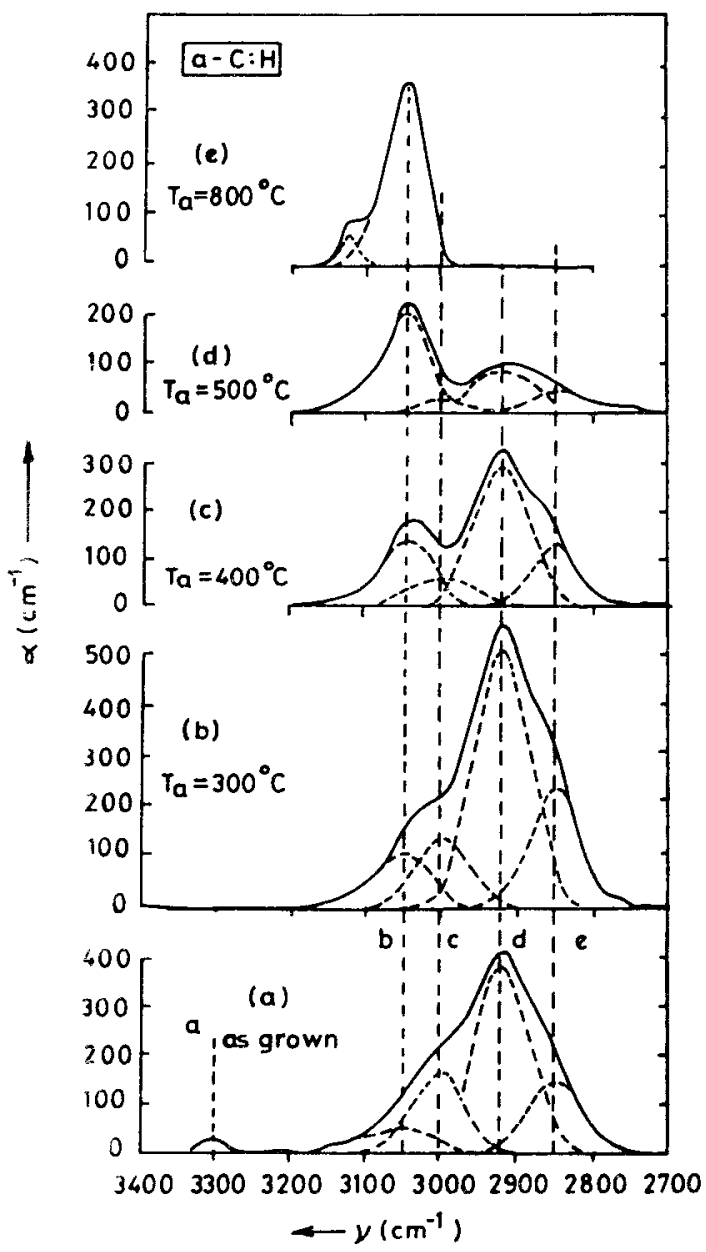

Figure 7 . Effect of heat treatment on the IR absorption spectrum of a typical DLC thin film. a. $s p^{1} \mathrm{CH}$, b. $s p^{2} \mathrm{CH}$ (arom.), c. $s p^{2} \mathrm{CH}$ (olef.), d. $s p^{3} \mathrm{CH}$ and e. $s p^{3} \mathrm{CH}_{2}$ (Dischler et al 1983). 


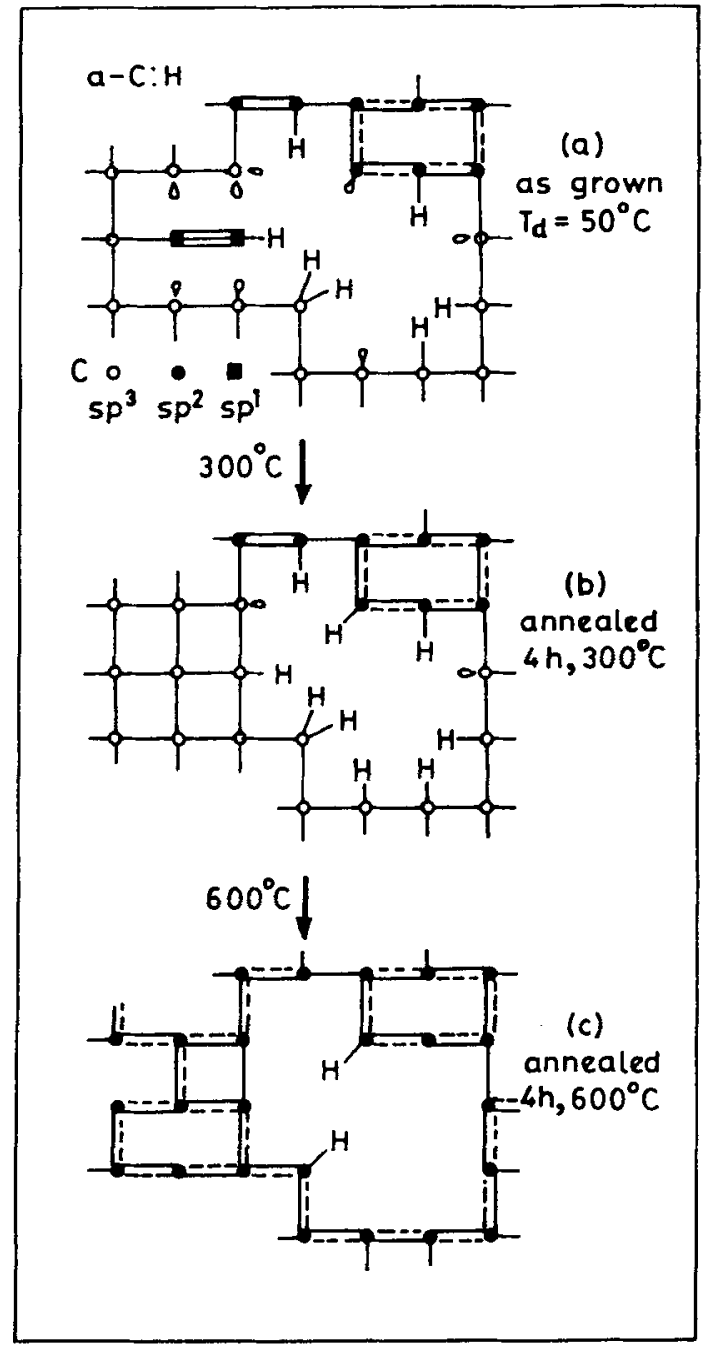

Figure 8. Proposed model for the thermally induced changes in the DLC microstructure. (a) as-grown, (b) after $300^{\circ} \mathrm{C}$ anneal and (c) after $600^{\circ} \mathrm{C}$ anneal (Dischler et al 1983).

paths $\left(10^{-1} \mathrm{ohm} \mathrm{cm}\right)$. Conducting pathways have thus been directly written in such films. Morphological changes suggest that most of the original DLC character is retained after laser irradiation of these films.

Considerable amount of hydrogen is also released during ion irradiation ( $\mathrm{KeV}$ to $\mathrm{MeV}$ range) of DLC films (Barshilia et al 1995). The associated microstructural changes are found to be related to defect formation in the films during the irradiation (Adel et al 1989).

The microstructure of DLC films has been determined by several workers using IR, Raman, NMR, EELS, AES and other techniques. Optical properties have been used quite extensively to determine the amount of $s p^{3}$ and $s p^{2}$ bonds in these films (Demichelis et al 1992 ; Fuchs et al 1993). Ramsteiner and Wagner (1987) have used resonant Raman scattering to show the existence of $n$-bonded clusters in DLC films. 
Tamor et al (1989) have shown the presence of pendant (mono-substituted) benzene groups in DLC films obtained from benzene source and this has been suggested to be the reason for a weak connection between the optical gap and the hydrogen content in the DLC films. Tamor and $\mathrm{Wu}(1990)$ have proposed a defective graphite model which ties together many important properties of DLC films containing varying amounts of hydrogen. Although this model is simple, it explains why optical properties of DLC films with wide variation in hydrogen content are remarkably similar, despite considerable variation in their structure.

To summarize we find that hydrogen plays a very important role in the nucleation and growth of microcrystalline diamond thin films. The microstructure of diamond thin films depends very critically on the surface and gas phase reactions involving hydrogen, which is present in very large concentrations in the deposition process. On the other hand incorporation of varied concentration of hydrogen may lead to DLC films with widely different microstructures which have a large combination of physical properties. The microstructural models proposed to explain these physical properties are based on the possible nature of hydrogen present in the films.

\section{Acknowledgement}

The author is very much grateful to the Department of Science and Technology, New Delhi for financial support.

\section{References}

Adel M E, Amir O, Kalish R and Feldman L C 1989 J. Appl. Phys. 663248

Angus J C, Koidl P and Domitz S 1986 in Plasma deposited thin films (Ed) J Mort and F Jansen (CRC Press Inc) p. 89

Barshilia H C, Sah S, Mehta B R. Vankar V D, Avasthi D K, Jaipal and Mehta G K 1995 Thin Solid Films 258123

Couderc P and Catherine Y 1987 Thin Solid Films 14693

Demichelis F, Pirri C F and Tagliaferro A 1992 Phys. Rev. B45 14364

Dischler B, Bubenzer A and Koidl P 1983 Solid State Commun. 48105

Frenklach M $1989 \mathrm{~J}$. Appl. Phys. 655142

Frenklach M and Spear K E 1988 J. Mater. Res. 3133

Fuchs A, Scherer J, Jung K and Ehrhardt H 1993 Thin Solid Films 23251

Hernandez J G. Chao B S and Pawlik D A 1989 J. Vac. Sci. \& Technol. A7 2332

Huang D, Frenklach M and Maroncelli M 1988 J. Phys. Chem. 926379

Inspector A, Mckenna T, Liou Y, Bourget L, Spear K E and Messier R 1989 in Diamond and diamond like films (eds) J P Dismukes et al (Pennington, NJ: The Electro Chem. Soc.) p. 342

Nadler M P, Donoven T M and Green A K 1984 Thin Solid Films 116241

Nyaiesh A R and Nowak W B 1983 J. Vac. Sci.\& Technol. A1 308

Pate B B, 1986 Surf. Sci. 16583

Pepper S V 1982 J. Vac. Sci.\& Technol. 20213

Prawer S, Kalish R and Adel M 1986 Appl. Phys. Lett. 485185

Ramsteiner M, Wagner J 1987 Appl. Phys. Lett. 511355

Robertson J 1986 Adv. Phys. 35317

Rossi F 1992 Advanced techniques for surf. engg. (eds) W Gissler and H A Jehn (Brussels: ECSE) p. 371

Setaka N 1989 J. Mater. Res. 4664

Sommet M, Mui K and Smith F W 1989 Solid State Commun. 69775

Spear K E 1989 J. Am. Ceram. Soc. 72171

Struck L M, D'Evelyn M P 1993 J. Vac. Sci.\& Technol. A11 
Tamor M A and Wu C H 1990 J. Appl. Phys. 671007

Tamor M A. Wu C H. Carter III R O and Lindsay N E 1989 Appl. Phy's. Lett. 551388

Tsai H C and Bogy D B 1987 J. Vac. Sci.\& Technol. A5 3287

Tsuda M. Nakajima M and Oikawa S $1986 \mathrm{~J}$. Am. Chem. Soc. 1085780

Vandenbulcke L. Bou P and Moreau G 1991 J. Electrochem. Soc. 1382985

Yang Y L, Struck L M, Sutchu L F and D'Evelyn M P 1993 Thin Solid Films 225203

Yarbrough W A and Messier R 1990 Science 247688 